\title{
Atypical Hypertrophic Osteoarthropathy as a Presenting Complaint in a Non-Smoker
}

\author{
Mark Robert Openshawa, ${ }^{\mathrm{a}}$, Clare Scarlett Rowan ${ }^{\mathrm{a}}$, Simon Grumett ${ }^{\mathrm{a}}$
}

\begin{abstract}
A 70-year-old non-smoker initially presented to accident and emergency with a painful left foot. This was an atypical presentation of hypertrophic osteoarthropathy (HPOA) secondary to grade 4 adenocarcinoma of the lung, highlighting the importance of routine chest $\mathrm{x}$-ray in clinical assessment of atypical bone pain. Diagnosis was made using an isotope bone scan, the most sensitive method for detecting HPOA. The tumour and HPOA symptoms responded well to the first line chemotherapy regimen of cisplatin and pemetrexed. This supports the current understanding that treatment of the primary lung lesion will treat HPOA. The mechanism of this action is hypothesised to be by reduction of endogenous growth factor production, either by reduction in cell numbers producing the factor or direct inhibition of factor production: VEGF is emerging as the predominant growth factor causing HPOA. We believe the mechanism of cisplatin action on HPOA symptoms may be two fold; firstly, by direct reduction in VEGF secreting tumour bulk and secondly, by reduction of VEGF transcription. After disease progression of the lung tumours and recurrence of HPOA symptoms the patient received second line docetaxel. HPOA symptoms were unchanged but the chemotherapy stabilised the tumours. This lack of effect on HPOA symptoms may be due to a lack of tumour reduction or more speculatively the lack of suppression of VEGF transcription. HPOA symptoms were significantly improved when the patient was started on octreotide and current studies suggest that VEGF inhibition may be the mechanism of octretotide's effect.
\end{abstract}

Keywords: Hypertrophic osteoarthropathy; Octreotide; Lung cancer; Vascular endothelial growth factor

Manuscript accepted for publication January 3, 2013

${ }^{a}$ Oncology Department, Russells Hall Hospital, Dudley, DY1 2HQ, United Kingdom

${ }^{\mathrm{b}}$ Corresponding author: Mark Robert Openshaw, Tauranga hospital, Cameron road, Tauranga, 3112, New Zealand.

Email: Mropenshaw@doctors.org.uk

doi: http://dx.doi.org/10.4021/jmc1057w

\section{Introduction}

Hypertrophic osteoarthropathy (HPOA) is an uncommon but well described complication of lung cancer. Rarely, as in this case, it may be a presenting feature of lung malignancy, even in people whom have never smoked. Medical practitioners should be aware that HPOA is a rare cause of non-resolving limb pain and may exhibit classic or atypical features. This case shows how symptoms of HPOA may respond to treatment of the underlying condition or to more direct therapies and we discuss the current theories regards its causes and treatments.

\section{Case Report}

A 70-year-old Caucasian man presented to the accident and emergency department with a first episode of significant left foot pain on standing. He was a lifelong non-smoker with a background of mild asthma, prostatic hypertrophy and depression. Following a medical assessment and podiatry review he was diagnosed with plantar fasciitis and treated with a course of physiotherapy.

Six weeks later he consulted his GP with a persistent non-productive cough, after seeing the local cancer awareness campaigns. Chest radiography revealed a $4 \mathrm{~cm}$ pulmonary nodule behind the apex of the heart and bilateral pulmonary nodules. CT showed a large right lower lobe mass with bilateral pulmonary nodules.

At 8 weeks his left foot had become increasingly painful and swollen. This was diagnosed as acute tendonitis and treated with immobilisation of the foot. Further examination showed no lymphadenopathy, clubbing, or pain involving the upper limbs. CT guided lung biopsy confirmed a stage 4 pulmonary adenocarcinoma. At review by the oncologist he was asymptomatic with regards to his chest. However he had an acutely tender and swollen left leg, with pain on weight bearing causing him to be wheelchair bound. Inflammatory markers and bone markers were normal at this point. Referral to orthopaedic surgeons was made to exclude osteomyelitis prior to chemotherapy for the lung malignancy. 


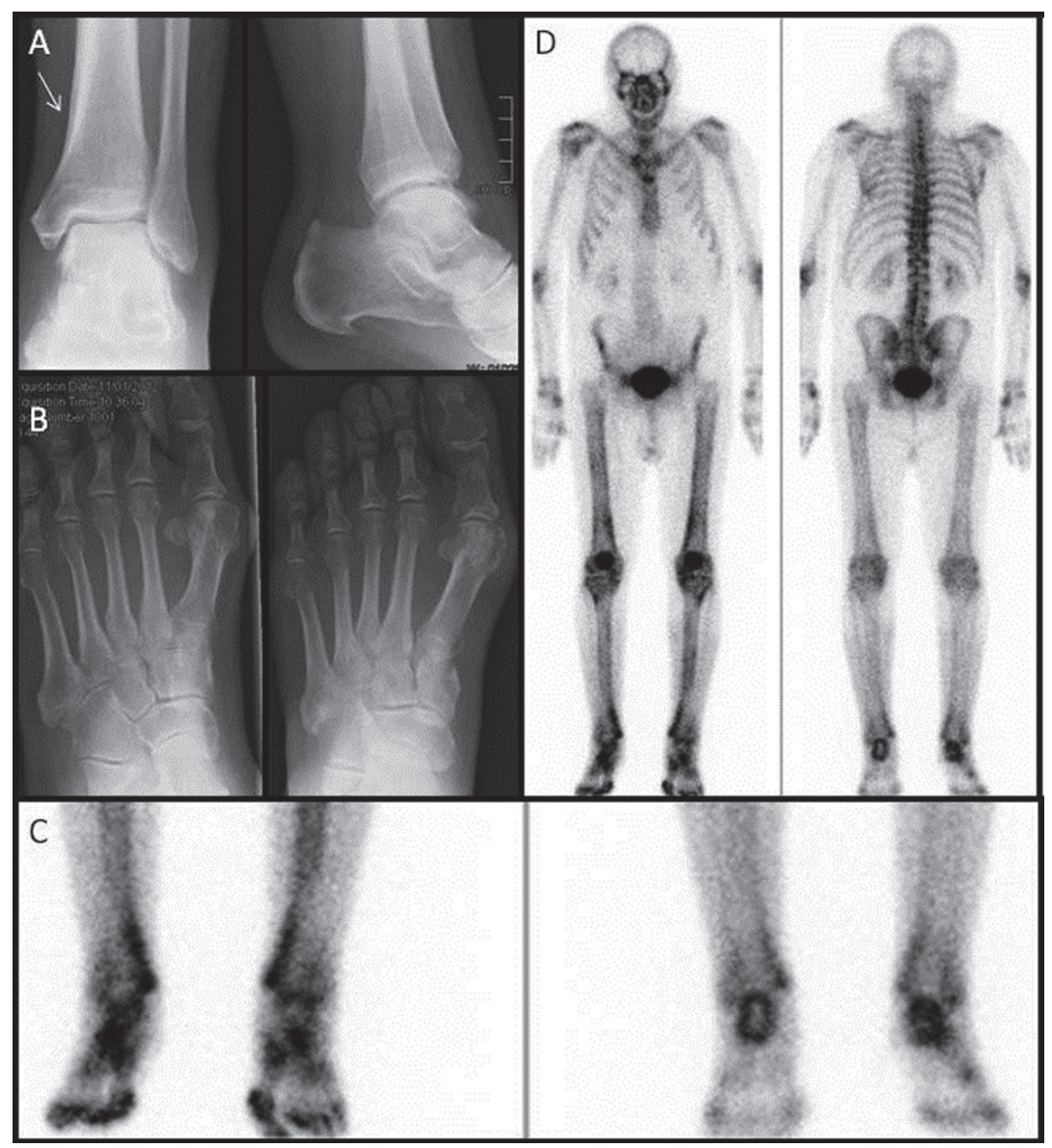

Figure 1. A) X-ray Left Ankle - periosteal reaction in the medial aspect of the distal left tibia; B) $X$-ray Left foot - Degenerative changes are noted in the tarsometatarsal joints and the first MTP joint. A calcaneal plantar spur is noted; $C$ and D) Isotope bone scan - linear increased uptake in the distal cortex of both tibias, particularly on the medial aspect. There is also some increased uptake in the cortex of the distal femoral shafts bilaterally.

Orthopaedic review reported pain on palpation of the left heel and over the ankle joint line, with mild pain in the right foot. Ankle radiography revealed a periosteal reaction in the medial aspect of the distal left tibia (Fig. 1A) whereas radiography of the left foot showed degenerative changes only (Fig. 1B). Ultrasound showed significant subcutaneous oedema but no evidence of plantar fasciitis or Achilles tendonitis. An isotope bone scan showed linear increased uptake in the distal cortex of both tibias and some increased uptake in the cortex of the distal femoral shafts bilaterally (Fig. 1C, D). The appearance was diagnostic of hypertrophic pulmonary osteoarthropathy (HPOA) affecting both legs secondary to carcinoma of the lung.

As biopsy analysis was negative for mutations in the $E G F R$ gene the patient was treated with pemetrexed and cisplatin chemotherapy. Following the first cycle, his leg pain and swelling had greatly improved allowing independent mobilisation. CT imaging after three cycles showed a good partial response. Repeat CT imaging after completion of 6 cycles showed disease progression but he remained free of chest symptoms. However, one month later his HPOA symptoms began to recur.

Docetaxel was elected for second line therapy. This resulted in stabilisation of the lung tumours but did not help to control his HPOA symptoms. However, the symptoms were partially alleviated by a successful trial of octreotide.

\section{Discussion}

Hypertrophic pulmonary osteoarthropathy (HPOA) was first described in 1889 [1] and can be split into primary (rare familial autosomal disease) and secondary causes. Secondary HPOA, also known as Pierre Marie-Bamberger syndrome accounts for $95 \%$ of cases [2]. Classically HPOA presents bilaterally and is described as a clinical triad of digital clubbing, ossifying periostitis of the long bones and arthralgia [3]. By contrast, this presentation was unilateral, and club- 
bing was absent, highlighting that other clinical symptoms such as constant pain, oedema and erythema in the extremities, are important diagnostic feature. HPOA is now widely agreed to be a paraneoplastic syndrome occurring in less than $1 \%$ of lung cancer patients but has been associated with other causes such as cystic fibrosis, cyanotic heart disease, and GI tract neoplasms [2]. It is related to a primary lung cancer in $80 \%$ of patients [4], and of these over $50 \%$ are caused by an adenocarcinoma [2]. HPOA can be a presenting feature and previous case reports have suggested chest radiography in all smokers and ex-smokers who present with synovitis [3]. This case demonstrates that it may be a presenting feature in non-smokers with lung cancer, highlighting the importance of routine chest $\mathrm{x}$-ray in clinical assessment of atypical bone pain and synovitis.

Diaphyseal and metaphyseal periostitis is the radiological hallmark of HPOA on plain film, causing periosteal thickening and new bone growth [5]. Bone scintigraphy is the investigation of choice, revealing changes earlier and with a higher sensitivity than plain radiography $[6,7]$. Single-photon emission computed tomography may increase the confidence of diagnosis [8].

The cause of HPOA remains poorly understood, but is thought to be due to the production of growth factors, such as growth hormone, oestrogen, adrenocorticotrophin or vascular endothelial growth factor (VEGF) by the lung lesion itself, or failure of the normal removal of these factors by pulmonary arteriovenous shunting $[2,7,9]$. Shunting is thought to be dominant factor in non-cancerous lesions but may also play a role in lung cancers due to intratumour shunting. There is growing evidence that VEGF may play a central role in HPOA. Firstly, it is a powerful endothelial cell-stimulator factor and VEGF receptors are found in subperiosteal bone cells. Secondly, elevated levels have been found in many diseases associated with HPOA and it is known to be produced by many tumours as well as in response to hypoxia. Finally, VEGF is a platelet derived factor and platelet dysfunction in arteriovenous shunting is thought to be involved in the pathogenesis of HPOA [10].

Treatment of the lung condition, as in this case, is hypothesised to exert its effect by the reduction in the number of cells producing endogenous growth factor, although the degree of response to treatment is sometimes incomplete or absent. There is evidence that cisplatin and other chemotherapy agents such as doxorubicin directly reduce VEGF transcription in both hypoxic situations and at baseline levels suggesting an alternative pathway for the action of chemotherapy on HPOA [11]. In contrast, docetaxel has not been shown to have any action on VEGF transcription [11], and the failure to provide symptomatic relief in this case may be a reflection of the lack of action on transcription in addition to failure to reduce tumour bulk.

The successful use of octreotide to provide pain relief in HPOA was first described by Johnson et al [12] in 1997 in a case of bronchial squamous cell carcinoma, but has since been reported in other causes of HPOA [13]. The mechanism of octreotide is yet to be fully elucidated. It may have an intrinsic non-opioid analgesic effect or be related to the inhibition of an unknown hormone [14]. However more recently somatostatin analogues including octreotide have been shown to inhibit the production of VEGF [14]. This suggests that the inhibition of VEGF may be the reason for the effect of octreotide on HPOA and that more potent inhibitors of VEGF such as the anti-VEGF monoclonal antibody bevacizumab may have a role in the treatment of HPOA [13, 15]. However to date we can find no reports of the effect of bevacizumab on the symptoms of HPOA in patients with lung cancer who have received the antibody as part of their anti-cancer therapy.

\section{Grant Support}

Nil.

\section{Declaration}

The study has not been presented in elsewhere.

\section{Conflict of Interest}

Nil.

\section{References}

1. Bamberger E: In Protokoll der k.k. Gesellschaft der Aertzte in Wein. Wiener KlinischeWochenschrift. 1889;2:226.

2. Ito T, Goto K, Yoh K, Niho S, Ohmatsu H, Kubota K, Nagai K, et al. Hypertrophic pulmonary osteoarthropathy as a paraneoplastic manifestation of lung cancer. J Thorac Oncol. 2010;5(7):976-980.

3. Armstrong DJ, McCausland EM, Wright GD. Hypertrophic pulmonary osteoarthropathy (HPOA) (Pierre Marie-Bamberger syndrome): two cases presenting as acute inflammatory arthritis. Description and review of the literature. Rheumatol Int. 2007;27(4):399-402.

4. Coury C. Hippocration fingers and hypertrophic osteoarthropathy. A study of 350 cases. Br J Dis Chest. 1960;54:202-209.

5. Sato K, Iwasaki Y, Kobayashi G, Sunaga N, Ishihara S, Watanabe S, Tuchiya S, et al. [Pulmonary hypertrophic osteoarthropathy associated with primary lung cancer]. Nihon Kokyuki Gakkai Zasshi. 2000;38(1):73-77.

6. Morgan B, Coakley F, Finlay DB, Belton I. Hypertro- 
phic osteoarthropathy in staging skeletal scintigraphy for lung cancer. Clin Radiol. 1996;51(10):694-697.

7. Uchisako H, Suga K, Tanaka N, Nishigauchi K, Matsumoto T, Matsunaga N, Nakanishi T. Bone scintigraphy in growth hormone-secreting pulmonary cancer and hypertrophic osteoarthropathy. J Nucl Med. 1995;36(5):822-825.

8. Lin Y, Tsai SC, Wang H, Lu Y, Lin W. The Usefulness of Bone Scintigraphy in the Evaluation of Hypertrophic Osteoarthropathy after Chemotherapy: A Case Report and Literature Review Ann Nucl Med Sci. 2010; 23(1):47-51.

9. Marino WD, Harigopalan JA, Bangar M. A 49-year-old smoker with a lung mass and diffuse bone pain. Chest. 2011;139(2):460-463.

10. Olan F, Portela M, Navarro C, Gaxiola M, Silveira LH, Ruiz V, Martinez-Lavin M. Circulating vascular endothelial growth factor concentrations in a case of pulmonary hypertrophic osteoarthropathy. Correlation with disease activity. J Rheumatol. 2004;31(3):614-616.

11. Duyndam MC, van Berkel MP, Dorsman JC, Rockx
DA, Pinedo HM, Boven E. Cisplatin and doxorubicin repress Vascular Endothelial Growth Factor expression and differentially down-regulate Hypoxia-inducible Factor I activity in human ovarian cancer cells. Biochem Pharmacol. 2007;74(2):191-201.

12. Johnson SA, Spiller PA, Faull CM. Treatment of resistant pain in hypertrophic pulmonary osteoarthropathy with subcutaneous octreotide. Thorax. 1997;52(3):298299.

13. Angel-Moreno Maroto A, Martinez-Quintana E, Suarez-Castellano L, Perez-Arellano JL. Painful hypertrophic osteoarthropathy successfully treated with octreotide. The pathogenetic role of vascular endothelial growth factor (VEGF). Rheumatology (Oxford). 2005;44(10):1326-1327.

14. Penson RT, Rudd RM. Commentary: octreotide and hypertrophic pulmonary osteoarthropathy. Thorax. 1997;52(3):297-298.

15. Dasgupta P. Somatostatin analogues: multiple roles in cellular proliferation, neoplasia, and angiogenesis. Pharmacol Ther. 2004;102(1):61-85. 